\title{
Patentes mexicanas del Distrito Federal: caracterización por delegación y área tecnológica (2009-2012)
}

\author{
Nevid Israel Meza Rodríguez* \\ Gabriela Millán Quintero** \\ Miguel Ángel Pérez Angón**
}

Artículo recibido:

15 de enero de 2014.

Artículo aceptado: 13 de octubre de 2015.
Resumen

El objetivo de este trabajo es estudiar la dinámica de las patentes otorgadas en México a residentes mexicanos ubicados en el Distrito Federal. Se entiende en este trabajo que las patentes dan muestra de la capacidad inventiva y son un elemento que impulsa las actividades de innovación en las empresas. El estudio se realiza como un aporte al desarrollo de políticas públicas encaminadas a la protección intelectual y a la innovación. Se encuentra que el Distrito Federal concentra casi la mitad de las patentes otorgadas a residentes mexicanos; las patentes corresponden a un nivel de intensidad

* Doctorado en Ciencias con especialidad en Desarrollo Científico y Tecnológico para la Sociedad, Cinvestav-IPN. nmeza@cinvestav.mx; gabmillan@cinvestav.mx

**Departamento de Física, Cinvestav-IPN. mperez@fis.cinvestav.mx

INVESTIGACIÓN BIBLIOTECOLÓGICA, vol. 31, núm. 71, enero/abril, 2017, México, ISSN: 0187-358X pp. 181-200 
tecnológica media-alta y tienen una dinámica de crecimiento constante durante el periodo estudiado.

Palabras clave: Distrito Federal; Patentes; Análisis de patentes; Capacidad inventiva; Estadística de patentes.

\section{Abstract}

Mexican's Federal District Patents: Characterization by Delegation and Technological Area (2009-2012)

Nevid Israel Meza Rodríguez, Gabriela Millán

Quintero, and Miguel Ángel Pérez Angón

The aim of this work is to study the dynamics of patents granted in Mexico to Mexican residents of Mexico City, te former Federal District. This paper assumes that patents are indicators of inventiveness as well as an incentivizing element driving the innovation process within companies. The study aims to contribute to the field of public policy development, specifically with regard to protecting intellectual property and innovation. The study found that the Mexico City accounts for almost half of the patents granted to Mexican residents, and that these patents correspond to a medium-high level of technological intensity. Moreover, during the period under study, steady growth in the granting of patents was observed.

Keywords: Mexico City; Patents; Patent Analysis; Inventiveness; Patent Statistics.

\section{Introducción}

工 patente es un instrumento jurídico que protege las invenciones novedosas que son resultado de una actividad inventiva y son susceptibles a la aplicación industrial por un plazo de 20 años. ${ }^{1}$ La patente, por su finalidad como mecanismo de protección y por su capacidad de intercambio de valor, recaba información importante sobre las empresas, instituciones o personas 
que las utilizan. Así, los indicadores de patentes contienen información sensible para el análisis estadístico de la actividad inventiva. ${ }^{2}$

Según la OCDE y la OEPM: "La finalidad declarada del sistema de patentes es fomentar la invención y el progreso técnico facilitando un plazo temporal de exclusividad sobre la invención a cambio de su divulgación. Al ofrecer protección y exclusividad, la patente constituye un instrumento político cuya finalidad es alentar a los inventores a que inviertan en investigación y en el trabajo de innovación posterior que conseguirá que esas invenciones tengan una aplicación práctica” ${ }^{3}$

La patente es entonces un instrumento de apoyo tanto para la gestión tecnológica de las empresas e instituciones como para la difusión de la información tecnológica en la sociedad. La patente, como mecanismo de propiedad industrial, se estudia como muestra de "los resultados de la investigación científica con potencial de aplicación” permitiendo ser un mecanismo de protección y de gestión comercial. ${ }^{4}$ Narín, ${ }^{5}$ Wallin ${ }^{6}$ y Verbeek et al., han observado que las investigaciones bibliométricas suelen enfocarse en publicaciones científicas académicas, sin embargo, estas metodologías pueden aplicarse también para el análisis de las patentes.

La importancia de las patentes como mecanismo de medida fue enfatizada en 1996 en la conferencia "Blue Sky", de la OCDE, en la cual diferentes expertos identificaron a las patentes como una forma adecuada para observar las actividades de ciencia y tecnología dentro de los contextos económicos y políticos. ${ }^{8}$ Como resultado de la importancia de las patentes, dentro de esta conferencia, se generó el Manual de estadísticas de patentes de la OCDE. ${ }^{9}$ Sin embargo, la importancia que las patentes tienen para entender las dinámicas de innovación se ha mostrado en otros manuales y diferentes sistemas de indicadores a nivel mundial.

\footnotetext{
OCDE y OEPM, Manual de estadísticas de patentes de la OCDE (París: OCDE, 2009), 15-29.

Ibid., 23.

RICyT, Manual de Santiago (Buenos Aires, Argentina: RICyT, 2009).

F. Narin, "Patent Bibliometrics", Scientometrics 30, no. 1 (1994): 147-155.

J. Wallin, "Bibliometric Methods: Pitfalls and Possibilities", Basic Clinical Pharmacology and Toxicology 97, no. 5 (2005): 261-275.

7 Verbeek, et al., "Measuring Progress and Evolution in Science and Technology: The Multiple Uses of Bibliometric Indicators", International Journal of Management Reviews 4, no. 2 (2002): 179-211.

8 OCDE y OEPM, Manual de estadísticas..., 4.

9 Ibid., 4.
} 
Si bien las patentes son una medida adecuada sobre capacidades inventivas de la población, ${ }^{10}$ es necesario considerar que: la pertinencia de la propiedad industrial asociada a las patentes se relaciona directamente con los objetivos estratégicos desde el punto de vista comercial de las empresas, instituciones o particulares. Por lo tanto, existen sectores e intereses que no requieren protecciones de este tipo. ${ }^{11}$ Sin embargo, al considerarlas como paso intermedio entre la investigación, el desarrollo (I+D) y la innovación; se convierten en una forma de medida adecuada para caracterizar los segmentos que utilizan este mecanismo de propiedad industrial.

Existen diferentes estudios publicados en revistas de corriente principal sobre las patentes registradas en países desarrollados, como en Whittington y Smith, ${ }^{12}$ McMillan, ${ }^{13}$ Sugimoto et al.,${ }^{14}$ Toivanen y Suominen, ${ }^{15}$ pero muy pocos sobre patentes de Latinoamérica, según Barroso ${ }^{16}$ y Guzman-Chavez et al..$^{17}$ Trabajos como el de Rodríguez-Pose y Villarreal, ${ }^{18}$ Hernández y Díaz, ${ }^{19}$ Sánchez, García y Mendoza ${ }^{20}$ y Vilalta y Banda-Ortiz, ${ }^{21}$ desarrollan investigaciones regionales basadas en patentes en México y su importancia para incentivar el desarrollo innovador a nivel nacional. Igualmente la $\mathrm{OCDE}^{22}$ ha realizado estudios de patentes a nivel regional en México. Todos los estudios observan que la concentración de las patentes en las regiones tiene repercusiones importantes en las entidades vecinas y que el Distrito Federal es la entidad más fuerte con respecto a la solicitud y concesión de patentes.

10 Ibid., 4

11 RICyT, Manual de Santiago.

12 K. Whittington y L. Smith, “Gender and Comercial Science: Women's Patenting in the Life Sciences”, Journal of Technology Transfer 30 (2005): 355-370.

13 G. S. McMillan, "Gender Differences in Patenting Activity: An Examination of U.S. Biotechnology Industry”, Scientometrics 10 (2009): 683-691.

14 C. R. Sugimoto et. al., "The Academic Advantage: Gender Disparities in Patenting", Plos One 10 , no. 5 (2015).

15 H. Toivanen y A. Suominen, "The Global Inventor Gap: Distribution and Equality of World-wide Inventive Efforts, 1990-2010”, Plos One 10, no. 4 (2005).

16 W. Barroso y L. A. Quoniam y E. Pacheco, "Patents as Technological Information in Latin America”, World Patent Information 31, no. 3 (2009): 207-215.

17 A. G. Guzman Chávez, F. Lopez-Herrera y F. Venegas Martínez, "Un análisis de co-integración entre patentes y crecimiento económico en México 1980-2008”, Investigación Económica 71 (2012): 83-115.

18 A. Rodríguez-Pose y E. Villarreal, "Innovation and Regional Growth in Mexico 2000-2010", Growth and Change 46, no. 2 (2015): 172-195.

19 S. Hernández y E. Díaz, "La producción y el uso del conocimiento en México y su impacto en la innovación: análisis regional de las patentes solicitadas”, Análisis Económico 50, no. 22 (2007): 185-217.

20 Y. Sánchez, F. García y E. Mendoza, "Determinantes de la capacidad de innovación regional en México: Una tipología de las regiones”, Región y Sociedad 26, no. 61 (2014).

21 C. Vilalta y H. Banda-Ortiz, "Two Hypothesis Tests for the Geography of Patents in Mexico", Economía, Sociedad y Territorio 8, no. 28 (2008): 1007-1032.

22 "Regional Concentration of Patents", en OCDE Regions at a Glance 2009 (OCDE, 2009), consultado en agosto de 2013, http://dx.doi.org/10.1787/reg_glance-2009-7-en. 
En este trabajo estudiamos la dinámica de crecimiento de las patentes otorgadas por el Instituto Mexicano de la Propiedad Industrial (IMPI) a residentes mexicanos con ubicación en el Distrito Federal (D. F.). En particular, nos interesa estudiar la distribución geográfica de los autores de las patentes por delegación y por área tecnológica de registro. Como ha sido el caso de la producción científica mexicana, ${ }^{23}$ nuestros resultados indican que el D. F. concentra casi la mitad de las patentes otorgadas a residentes mexicanos y estas patentes se ubican en un nivel de intensidad tecnológica media-alta.

\section{Metodología}

Para el desarrollo de este trabajo se utilizaron las bases de patentes SIGA ${ }^{24}$ del IMPI y el segmento del Distrito Federal proporcionado por la Dirección General de Evaluación Institucional de la Universidad Nacional Autónoma de México DGEI-UNAM. ${ }^{25}$ La integración de información se realizó con las patentes otorgadas a residentes nacionales (por nacionalidad del titular MX). Para acotar la información a las patentes otorgadas en el Distrito Federal se identificó la dirección del titular (entidad Distrito Federal). Para realizar la distribución por delegación política se identificaron los códigos postales ${ }^{26}$ de la dirección del titular de cada patente. Se homologaron los datos de titulares y se clasificaron en: empresas, particulares, instituciones de educación superior e institutos y centros de investigación; posteriormente se reconocieron los vínculos de cotitularidad entre los participantes de las patentes. Se distinguieron las principales Clasificaciones Internacionales de Patentes (CIP) que participan dentro de las invenciones; se categorizaron según el área tecnológica y nivel tecnológico. Se filtraron y homologaron las principales palabras para un análisis de títulos y finalmente se registraron las patentes internacionales por medio del número de solicitud del PCT. La información es complementada con datos recabados de diferentes documentos y bases de datos como: Atlas de la Ciencia Mexicana, ${ }^{27}$ IMPI, ${ }^{28}$ Conacyt $^{29}$

23 Atlas de la ciencia mexicana, 2012, consultado en marzo de 2014, http://www.atlasdelaciencia mexicana.org.mx.

24 Sistema de información de la Gaceta de la Propiedad Industrial (México: Instituto Mexicano de la Propiedad Industrial, 2014), http://siga.impi.gob.mx.

25 "Estudio comparativo de las universidades mexicanas" (México: UNAM, EXECUM, 2013), consultado en marzo de 2014. http://www.execum.unam.mx/index2.php?_dc=0.

26 SCT, Correos de México, Consulta Códigos Postales, marzo 2014, http://www.correos demexico.gob.mx/ServiciosLinea/Paginas/ccpostales.aspx.

27 Atlas de la ciencia mexicana, 2012

28 IMPI, IMPI en cifras (México: Instituto Mexicano de la propiedad Industrial, 2013).

29 Conacyt, Informe general del estado de la ciencia, la tecnología y la innovación, México: Consejo Nacional de Ciencia y Tecnología, 2012; Conacyt, Sistema integrado de información sobre investigación científica, desarrollo tecnológico e innovación, México: Consejo Nacional de Ciencia y Tecnología, consultado en marzo de 2014, http://www.siicyt.gob.mx/siicyt/. 
e INEGI..$^{30}$ El trabajo de redes se realiza con el programa Pajek en su versión 2.05.31

\section{Desarrollo}

El Distrito Federal comprende un territorio de $1485 \mathrm{~km}^{2}$ en el que se concentraron 8851080 habitantes durante 2010, que representa el 7.88\% de la población total de México. Durante 2009 el Distrito Federal tuvo una participación del $40.74 \%$ del total de investigadores del país. ${ }^{32}$ En la república Mexicana, durante el periodo 2009-2012, se solicitaron 58226 patentes, de las cuales 4130 fueron solicitudes de residentes mexicanos. De éstas $4130 \mathrm{pa}-$ tentes, se otorgaron 968 , donde el $44 \%$ corresponde a residentes de el Distrito Federal. La participación de el Distrito Federal es la más importante de las participaciones en éste periodo de tiempo con respecto a las demás entidades federativas. En la Figura 1 se puede observar la participación que tiene la entidad con relación al otorgamiento de patentes a residentes.

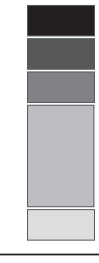

$43 \%$ Cdmx

$8 \%, 15 \%$ Méx, NL, Jal

$0.1 \%, 2 \%$ Qro, Mor, Pue, Gto, Coah, Chih

$0.1 \%, 2 \%$ Ver, San, Tam, Tab, Mich, Yuc, Ags, SLP, BC, Col, Sin, Oax, BCS,

Dgo, Hgo, Chis, Gro, QRoo, Tlax, Camp

$0 \%$, Nay, Zac

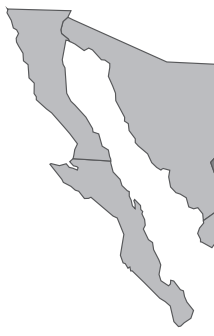

Figura 1. Distribución geográfica de las patentes otorgadas a residentes mexicanos (2009-2012) Fuente: Elaboración propia con datos de IMPI (2014) y UNAM (2013). ${ }^{33}$

30 INEGI, Instituto Nacional de Estadística y Geografía (México, 2014), consultado en marzo de 2014, http://www.inegi.org.mx/default.aspx.

31 V. Batagelj y A. Mrvar, Networks/Pajek64, Program for Large Network. Analysis, 2 mayo de 1996, consultado en enero de 2013, http://vlado.fmf.uni-lj.si/pub/networks/pajek/.

32 INEGI, 2014 y Atlas de la ciencia mexicana, 2012.

33 Todas los gráficos, las figuras y las tablas se realizaron como resultado del trabajo de investigación con los datos de IMPI, Sistema de información de la gaceta... y UNAM, "Estudio comparativo de las universidades mexicanas". Excepto para la Tabla 1. 
Como se puede observar en la Figura 1, el Distrito Federal concentra el $43 \%$ de las patentes otorgadas y los siguientes tres estados (Estado de México, Nuevo León y Jalisco) participan cada uno de ellos con un porcentaje que va del $8 \%$ al 15\%. Nayarit y Zacatecas no cuentan con patentes otorgadas en este periodo de tiempo.

Poniendo atención directamente al caso de el Distrito Federal, se puede identificar la tendencia histórica de las patentes distinguiendo un crecimiento constante de las actividades de registro en la entidad. La Figura 2 da muestra de este proceso histórico en el que se observa tanto la base del Instituto Mexicano de la Propiedad Industrial ${ }^{34}$ como la base proporcionada por la Dirección General de Evaluación Institucional de la Universidad Nacional Autónoma de México. ${ }^{35}$

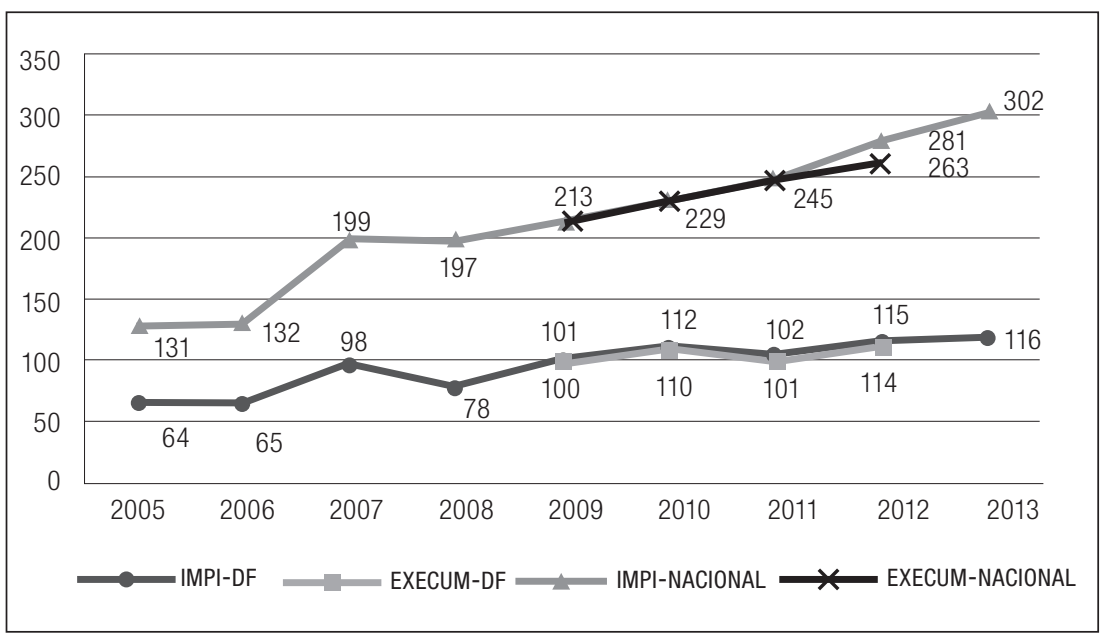

Figura 2. Evolución del número de patentes otorgadas a residentes mexicanos: comparación Ciudad de México y Nacional 2006-2012. Fuente: Elaboración propia con datos de IMPI (2014) y UNAM (2013).

Sobre la distribución de patentes por delegación en el Distrito Federal, podemos notar que siete delegaciones (Gustavo A. Madero, Miguel Hidalgo, Coyoacán, Benito Juárez, Tlalpan, Álvaro Obregón y Cuauhtémoc) concentran el $90.54 \%$ de las patentes concedidas en el periodo, mientras que en Milpa Alta no se registraron patentes. De las patentes otorgadas en el área que comprende el Distrito Federal, encontramos que el 33.33\% corresponden a patentes otorgadas a individuos independientes o particulares, el $24.02 \%$ a empresas, el $20.08 \%$ a institutos y centros de investigación,

34 IMPI, Sistema de información de la gaceta...

35 UNAM, "Estudio comparativo de las universidades mexicanas". 
el $18.01 \%$ a instituciones de educación superior, el 3.73\% a instituciones de salud y el $0.83 \%$ corresponde a instituciones de gobierno. Dicha segmentación de actores se hace bajo las concepciones del Triángulo de Sábato ${ }^{36}$ y de la Triple Hélice, ${ }^{37}$ que observan tres agentes principales dentro de los sistemas de innovación y los vínculos sobre las actividades que desempeñan individual y colectivamente. Así, estos modelos teóricos establecen una primera categorización de actores que intervienen en los procesos de innovación: universidades, empresas y gobierno.

\section{Análisis de cotitularidad}

De las patentes estudiadas dentro de este trabajo, únicamente el $8.98 \%$ estableció algún tipo de colaboración con alguna otro actor del sistema obteniendo así la cotitularidad en la patente. Por tanto, no se puede notar una relación funcional entre los actores siguiendo la dinámica de las concepciones del Triángulo de Sábato ${ }^{38}$ o de la Triple Hélice. ${ }^{39}$ Las colaboraciones que se dan son principalmente entre particulares (el $44.74 \%$ ), seguidas por los institutos de educación superior con empresas (el 28.95\%) o con institutos y centros de investigación (el 15.79\%). Se pueden observar dinámicas en las que los institutos de educación superior igualmente se relacionan con instituciones de salud, que a su vez están relacionados con institutos y centros de investigación.

Considerando a los titulares más representativos a los que se les concedió una patente durante el segmento de tiempo (Figura 3), se observa la importancia que tienen los institutos y centros de investigación y las instituciones de educación superior dentro del otorgamiento de patentes en el D.F. Sin bien se registró una aportación alta (el 33.33\%) de los individuos independientes o particulares dentro de este proceso, el papel de los centros de investigación sumado con el de las instituciones de educación superior lo supera en un $38.10 \%$. De las seis formas de interrelación de titularidad entre los participantes del segmento de tiempo, las formas más recurrentes fueron las que se dieron entre particulares y entre instituciones de educación superior y empresas.

36 J. Sábato y B. Natalio, "La ciencia y la tecnología en el desarrollo futuro de América Latina", Revista de Integración (1968).

37 H. Etzkowitz, A. Webster, C. Gebhardt y B. Cantisano, "The Future of the University and the University of the Future: Evolution of Ivory Tower to Entrepreneurial Paradigm”, Research Policy 29 (2000): 313-330.

38 Sábato y Natalio, "La ciencia y la tecnología en el desarrollo futuro de América Latina".

39 Etzkowitz, Webster, Gebhardt y Cantisano, "The Future of the University and the University of the Future...", 313-330. 


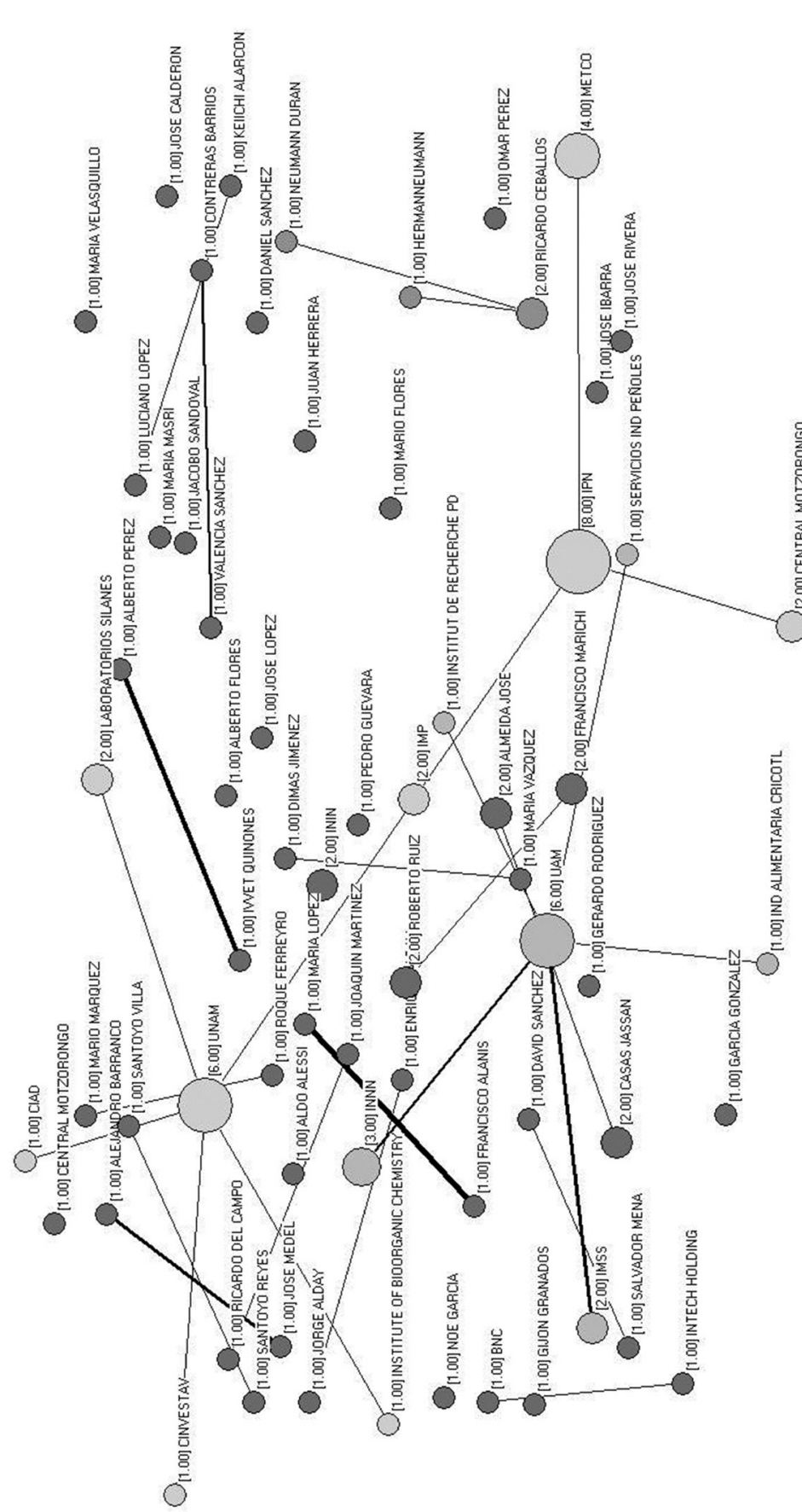

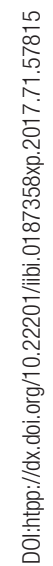




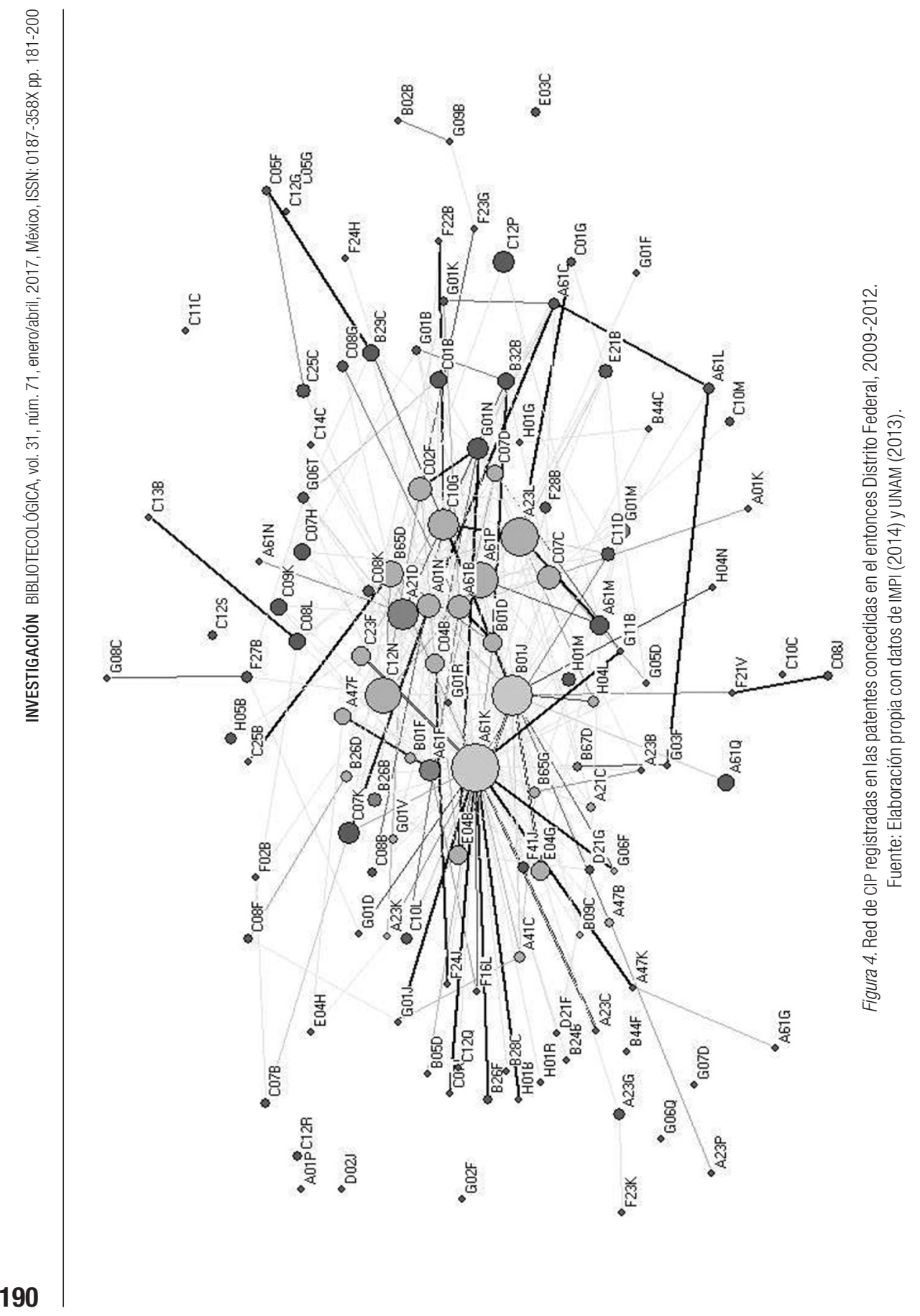




\section{Análisis de tendencias y nivel tecnológico}

Haciendo un estudio sobre la Clasificación Internacional de la Patente (CIP), podemos observar las tendencias tecnológicas y el nivel de intensidad tecnológicos. De forma inicial se estudió la CIP de la patente para identificar la frecuencia de participación de cada una de ellas en la dinámica, como se muestra en la Figura 4. Las primeras clasificaciones ocupan el 20\% de las patentes, y las once primeras clasificaciones agrupan el 50\% de la representatividad. Dichas clasificaciones son respectivamente: A61K, B01J, A23L, A61P, C12N, A21D, C10G, B65D, A61B, C02F y A01N (véase Tabla 1).

Tabla 1. Descripción de la Clasificación Internacional de Patentes (CIP) contenida en las patentes concedidas a residentes del Distrito Federal 2009-2012.

\begin{tabular}{|c|c|c|}
\hline CIP & Definición & Ejemplo \\
\hline A61K & Preparaciones de uso médico, dental o para el aseo. & $\begin{array}{l}\text { Composiciones } \\
\text { farmacéuticas }\end{array}$ \\
\hline B01J & $\begin{array}{l}\text { Procedimientos Químicos o Físicos, por ejemplo: catálisis, } \\
\text { química de los coloides; aparatos adecuados. }\end{array}$ & Catalizadores \\
\hline A23L & $\begin{array}{l}\text { Alimentos, productos alimenticios o bebidas no alcohólicas, } \\
\text { preparación o tratamiento, por ejemplo: cocción, modificación } \\
\text { de las cualidades nutricionales, tratamiento físico, conservación } \\
\text { de alimentos o de productos alimenticios, en general. }\end{array}$ & Productos alimenticios \\
\hline A61P & $\begin{array}{l}\text { Actividad terapéutica específica de compuestos químicos } \\
\text { o de preparaciones medicinales. }\end{array}$ & Productos farmacéuticos \\
\hline $\mathrm{C} 12 \mathrm{~N}$ & $\begin{array}{l}\text { Microorganismos o enzimas; compuestos que los contienen, } \\
\text { propagación, cultivo o conservación de microorganismos; } \\
\text { técnicas de mutación o de ingeniería genética; medios de cultivo. }\end{array}$ & Biotecnología \\
\hline A21D & $\begin{array}{l}\text { Tratamiento, por ejemplo: conservación de la harina o de la masa } \\
\text { por adición de ingredientes; cocción; productos de panadería; su } \\
\text { conservación. }\end{array}$ & Productos alimenticios \\
\hline C10G & $\begin{array}{l}\text { Cracking de los aceites de hidrocarburos; producción de mezclas } \\
\text { de hidrocarburos líquidos, por ejemplo: por hidrogenación } \\
\text { destructiva, por oligomerización y por polimerización, } \\
\text { recuperación de aceites de hidrocarburos a partir de esquistos, } \\
\text { de arena petrolífera o gases; refinamiento de mezclas } \\
\text { compuestas principalmente de hidrocarburos; reformado } \\
\text { de nafta; ceras minerales. }\end{array}$ & $\begin{array}{l}\text { Catalizadores de } \\
\text { hidrocarburos }\end{array}$ \\
\hline B65D & $\begin{array}{l}\text { Recipientes para el almacenamiento o el transporte de objetos } \\
\text { o materiales, por ejemplo: sacos, barriles, botellas, cajas, latas, } \\
\text { cartones, arcas, botes, bidones, tarros, tanques, accesorios } \\
\text { o cierres para recipientes; elementos de embalaje; paquetes. }\end{array}$ & Estantes y contenedores \\
\hline
\end{tabular}




\begin{tabular}{|c|c|c|}
\hline A61B & Diagnóstico; cirugía; identificación. & Instrumental médico \\
\hline $\mathrm{CO} 2 \mathrm{~F}$ & Tratamiento del agua, agua residual, de alcantarilla o fangos. & Plantas de tratamiento \\
\hline $\mathrm{A} 01 \mathrm{~N}$ & $\begin{array}{l}\text { Conservación de cuerpos humanos, animales, de vegetales } \\
\text { o de partes de ellos. biocidas, por ejemplo: en tanto sean } \\
\text { desinfectantes, pesticidas o herbicidas, productos que atraen } \\
\text { o repelen a los animales; reguladores del crecimiento de los } \\
\text { vegetales. }\end{array}$ & $\begin{array}{l}\text { Desinfectantes y } \\
\text { estimulantes vegetales }\end{array}$ \\
\hline
\end{tabular}

Fuente: Elaboración propia con información de OMPI. ${ }^{40}$

Según las recomendaciones de OCDE Y OEPM, ${ }^{41}$ OMPI $^{42}$ y RICyT, OEA y CyTED $^{43}$ la intensidad tecnológica asociada a una patente está definida por la clasificación internacional de patentes de cada patente. Lo que se observa es que 273 de las patentes del D. F. corresponden a un nivel tecnológico medio-alto, lo que representa el $64 \%$ del total de las concesiones otogadas en el Distrito Federal durante el periodo 2009-2012. 70 patentes se ubicaron en un nivel de alta tecnología, 60 abarcaron el nivel de media-baja tecnología y 20 patentes tienen un nivel de baja tecnología. La Figura 5 da muestra de la participación porcentual de dichos niveles tecnológicos.

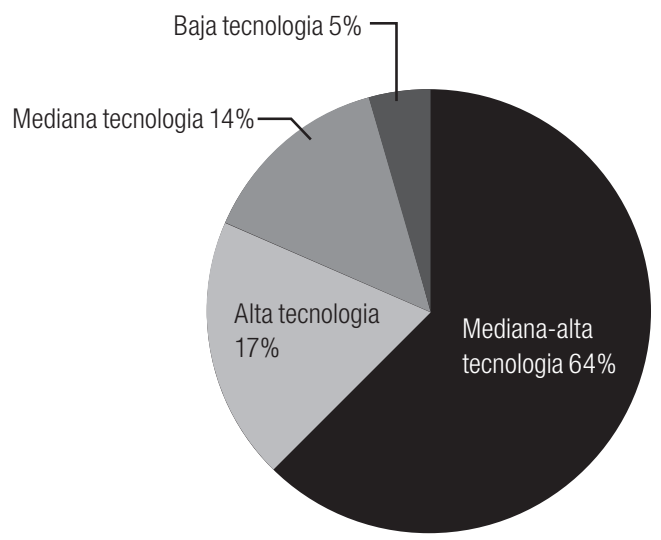

Figura 5. Participación porcentual de las patentes otorgadas a residentes del Distrito Federal 2009-2012 clasificadas según su nivel de intensidad tecnológico.

Fuente: Elaboración propia con datos de IMPI (2014) y UNAM (2013).

40 OMPI, “Clasificación Internacional de patentes”, 28 de febrero, 2014, consultado en marzo de 2014, http://cip.oepm.es/ipcpub/\#lang=es\&menulang=ES\&refresh=page.

41 OCDE y OEPM, Manual de estadísticas...

42 OMPI, Concept of a Technology Classification for Country Comparisons. World Intellectual Property Organization, 2008.

43 RICyT, OEA y CyTED, Manual de Bogotá, 2001. 
Con el fin de ponderar las patentes con un nivel de intensidad tecnológica alta se elabora un listado de las delegaciones según su participación en las patentes otorgadas a residentes del Distrito Federal 2009-2012. El orden del listado se muestra en la Tabla 2. Se puede notar que 14 de 16 delegaciones tienen una mayor participación de patentes con un nivel tecnológico medio-alto. No parece haber relación entre el principal actor patentador de cada delegación y el nivel tecnológico de las patentes observadas. Sin embargo, la participación de las diferentes instituciones favorece la cantidad de patentes otorgadas.

Tabla 2. Patentes de delegaciones según el nivel tecnológico y principales titulares*

\begin{tabular}{|c|c|c|}
\hline \multicolumn{3}{|c|}{ Gustavo A. Madero 122} \\
\hline \multicolumn{3}{|c|}{ Nivel tecnológico: Alta (19) Media-Alta (87) Media-Baja (16) Baja (0) } \\
\hline $\mathrm{IyCl}$ & CINVESTAV, IMPI & 91 \\
\hline IES & IPN & 21 \\
\hline Emp & Electroformados, Paniplus, Quinasa & 3 \\
\hline \multicolumn{3}{|c|}{ Miguel Hidalgo 62} \\
\hline \multicolumn{3}{|c|}{ Nivel tecnológico: Alta (9) Media-Alta (36) Media-Baja (12) Baja (5) } \\
\hline Emp & Laboratorios Senosiain, Sabritas, Power Retaling, Cementos Apasco, Accesos Holograficos & 35 \\
\hline IGob & Secretaría de la Defensa Nacional & 1 \\
\hline \multicolumn{3}{|c|}{ Coyoacán 54} \\
\hline \multicolumn{3}{|c|}{ Nivel tecnológico: Alta (14) Media-Alta (34) Media-Baja (6) Baja (0) } \\
\hline IES & UNAM & 39 \\
\hline Emp & Cell Therapy and Technology & 2 \\
\hline IGob & Secretaría de Marina-Armada de México & a \\
\hline \multicolumn{3}{|c|}{ Benito Juárez 40} \\
\hline \multicolumn{3}{|c|}{ Nivel tecnológico: Alta (11) Media-Alta (22) Media-Baja (1) Baja (6) } \\
\hline Emp & Farmaceuticos Rayere, Laboratorios Silanes, Biokab, Parfarm & 18 \\
\hline IES & Dirección General de Educación Superior Tecnológica, UNAM & 5 \\
\hline \multicolumn{3}{|c|}{ Tlalpan 39} \\
\hline \multicolumn{3}{|c|}{ Nivel tecnológico: Alta (7) Media-Alta (27) Media-Baja (5) Baja (0) } \\
\hline IES & UAM & 18 \\
\hline Emp & Laboratorios Dermatológicos Darier, Farmaceuticos Lakeside, Innovamedica & 8 \\
\hline
\end{tabular}

\footnotetext{
* En la tabla no aparece la participación de los particulares IyCI - Institutos y Centros de Investigación

IES - Instituciones de Educación Superior

Emp - Empresas

IGob - Instituciones de Gobierno

ISal - Instituciones de Salud
} 


\begin{tabular}{|c|c|c|}
\hline ISal & Instituto Nacional de Neurología y Neurocirugía, Instituto Nacional de Psiquiatría & 2 \\
\hline IGob & Instituto Electoral del Distrito Federal & 1 \\
\hline \multicolumn{3}{|c|}{ Álvaro Obregón 35} \\
\hline \multicolumn{3}{|c|}{ Nivel tecnológico: Alta (2) Media-Alta (19) Media-Baja (12) Baja (2) } \\
\hline Emp & Grupo Bimbo, Concretos translúcidos, 3M México, Indorama Ventures Polymers México & 21 \\
\hline IES & Universidad Iberoamericana & 1 \\
\hline \multicolumn{3}{|c|}{ Cuauhtémoc 31} \\
\hline \multicolumn{3}{|c|}{ Nivel tecnológico: Alta (4) Media-Alta (20) Media-Baja (1) Baja (6) } \\
\hline ISal & Instituto Mexicano del Seguro Social & 11 \\
\hline Emp & Naturismo éxito y salud, Biotecnología Orgánica, Frederick International & 8 \\
\hline IGob & Instituto Mexicano del Transporte & 1 \\
\hline \multicolumn{3}{|c|}{ Iztapalapa 14} \\
\hline \multicolumn{3}{|c|}{ Nivel tecnológico: Alta (0) Media-Alta (13) Media-Baja (1) Baja (0) } \\
\hline Emp & Calentadores de América, Laboratorio Avi-Mex, Square d Company México & 4 \\
\hline \multicolumn{3}{|c|}{ Magdalena Contreras 6} \\
\hline \multicolumn{3}{|c|}{ Nivel tecnológico: Alta (1) Media-Alta (3) Media-Baja (2) Baja (0) } \\
\hline \multicolumn{3}{|c|}{ Cuajimalpa de Morelos 5} \\
\hline \multicolumn{3}{|c|}{ Nivel tecnológico: Alta (1) Media-Alta (1) Media-Baja (3) Baja (0) } \\
\hline Emp & Macro-M, Sucroliq & 2 \\
\hline \multicolumn{3}{|c|}{ Iztacalco 5} \\
\hline \multicolumn{3}{|c|}{ Nivel tecnológico: Alta (0) Media-Alta (3) Media-Baja (1) Baja (1) } \\
\hline Emp & Tover cerraduras de México, S.A. de C.V. & 1 \\
\hline \multicolumn{3}{|c|}{ Azcapotzalco 4} \\
\hline \multicolumn{3}{|c|}{ Nivel tecnológico: Alta (2) Media-Alta (2) Media-Baja (0) Baja (0) } \\
\hline Emp & TD Vision Corporation, Optar productos y servicios & 3 \\
\hline \multicolumn{3}{|c|}{ Venustiano Carranza 3} \\
\hline \multicolumn{3}{|c|}{ Nivel tecnológico: Alta (0) Media-Alta (3) Media-Baja (0) Baja (0) } \\
\hline \multicolumn{3}{|c|}{ Xochimilco 2} \\
\hline \multicolumn{3}{|c|}{ Nivel tecnológico: Alta (0) Media-Alta (2) Media-Baja (0) Baja (0) } \\
\hline \multicolumn{3}{|c|}{ Tláhuac 1} \\
\hline \multicolumn{3}{|c|}{ Nivel tecnológico: Alta (0) Media-Alta (1) Media-Baja (0) Baja (0) } \\
\hline \multicolumn{3}{|c|}{ Milpa Alta 0} \\
\hline & Iente: Elaboración propia con datos de IMPI (2014) y UNAM (2013). & \\
\hline
\end{tabular}




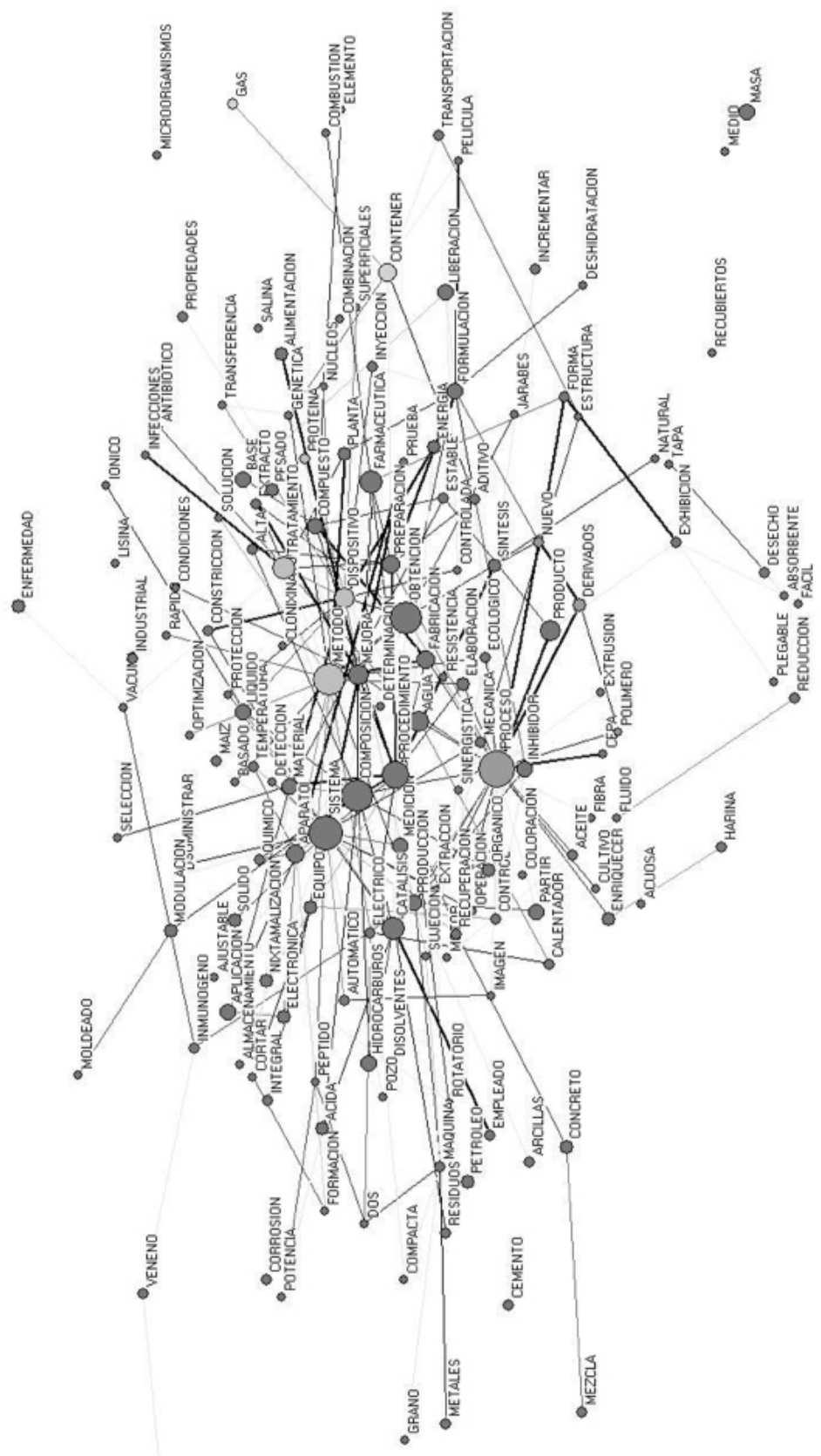

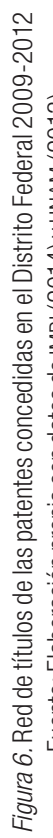

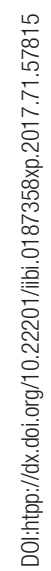

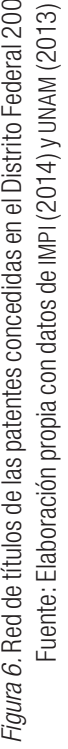




\section{Análisis de títulos}

El análisis de los títulos de las patentes nos permite reconocer los temas dentro de los que se están desarrollando avances tecnológicos. Para ello, se consideran las palabras principales de cada título y se observa su relación. La Figura 6 da muestra de esta dinámica. Como se puede observar en la red de títulos, las palabras más recurrentes nos hablan sobre el tipo de patente (producto o proceso). Las siguientes palabras nos hablan de los temas más recurrentes. En los títulos tenemos: farmacéutica (24), catálisis (23), agua (21), aparato (17), fabricación (15), hidrocarburos (14), base (13), formulación (13), material (13), medición (13), compuesto (12), crudo (12), inhibidor (12), liberación (12), líquido (11), masa (11), partir (11), derivados (10), pesado (10), síntesis (10).

\section{Análisis de patentes internacionales}

Entre las patentes revisadas 49 son internacionales, lo que equivale al $11.58 \%$ del total de las patentes otorgadas a residentes del Distrito Federal en el periodo 2009-2012. Del total de las patentes internacionales, el 48.98\% corresponde a patentes de empresas. Dentro de esta dinámica le siguen los particulares con un $32.65 \%$, instituciones de educación superior con un $12.24 \%$ y finalmente institutos y centros de investigación con un $6.12 \%$. Los principales titulares de estas patentes internacionales se muestran en la Tabla 3.

Tabla 3. Principales titulares de patentes internacionales residentes del Distrito Federal.

\begin{tabular}{|l|c|c|}
\hline \multicolumn{1}{|c|}{ Titulares } & N. de patentes & Porcentaje \\
\hline Empresa & 24 & $48.98 \%$ \\
\hline Sabritas, S. de R. L. de C. V. & 8 & \\
\hline Farmacéuticos Rayere, S.A. & 4 & \\
\hline Indorama Ventures Polymers México, S. de R.L. de C.V. & 2 & \\
\hline Laboratorios Silanes, S.A. de C.V. & 2 & \\
\hline Td Vision Corporation S.A. de C.V. & 2 & \\
\hline Particular & 16 & $32.65 \%$ \\
\hline Casas Jassan, Genaro & 2 & \\
\hline Savoir, John Claude & 2 & \\
\hline Instituciones de educación superior & 6 & $12.24 \%$ \\
\hline Universidad Nacional Autónoma de México & 5 & \\
\hline
\end{tabular}




\begin{tabular}{|l|l|l|}
\hline Universidad Iberoamericana, A.C. & 1 & \\
\hline Institutos y centros de investigación & 3 & $6.12 \%$ \\
\hline Instituto Mexicano del Petróleo & 2 & \\
\hline Centro de Investigación y de Estudios Avanzados del IPN. & 1 & \\
\hline \multicolumn{2}{|c|}{ Fuente: Elaboración propia con datos de IMPI (2014) y UNAM (2013). } \\
\hline
\end{tabular}

\section{Conclusiones}

El análisis regional de patentes permite hacer una revisión adecuada de la situación de los participantes, las tendencias y los niveles de intensidad tecnológicos en una zona geográfica determinada. En el Distrito Federal se concentra casi la mitad de las patentes otorgadas a residentes mexicanos. Este efecto nos habla de dos rubros importantes: de las características de esta entidad para el desarrollo de procesos y capacidades inventivas y del papel preponderante que juegan los inventores de esta entidad en el proceso de innovación regional y nacional.

La dinámica de patentamiento en el Distrito Federal se concentra principalmente en siete delegaciones donde la titularidad se puede clasificar (por orden de participación) en particulares, empresas, instituciones y centros de investigación, instituciones de educación superior, instituciones de salud e instituciones de gobierno. La residencia del solicitante, en este caso la delegación en la que se tramita la protección, nos permite tener un punto de partida sobre el mercado y las capacidades de investigación y desarrollo localizadas en las diferentes demarcaciones. La correcta ubicación de los participantes, así como los elementos de contexto que sirven como incentivos para el desarrollo de patentes son elementos útiles para fomentar una vinculación adecuada entre los actores de este proceso de innovación. Así, el reconocimiento de los titulares de las patentes de una entidad es una muestra aproximada del rendimiento inventivo de las instituciones.

El análisis de la Clasificación Internacional de Patentes (CIP) mostró que los rubros de desarrollo tecnológico principalmente se encuentran en las clasificaciones relacionadas con: necesidades corrientes de la vida, técnicas industriales diversas, así como, transporte y química y metalurgia. Los títulos permiten observar, igualmente, que las áreas de interés local se encuentran en torno a tecnologías de farmacéutica, catálisis, agua, entre otras. Reconocer la importancia de estas áreas para el desarrollo de invenciones puede ser un mecanismo adecuado para la construcción de políticas públicas dirigidas a proporcionar incentivos a tecnologías específicas. 
Además, constituyen una herramienta adecuada para propiciar las transferencias de tecnología y las actividades de vinculación académica entre aquellos interesados en el desarrollo de tecnologías semejantes.

Las patentes del Distrito Federal muestran un nivel de intensidad tecnológico medio-alto. Lo cual nos habla de las capacidades locales para el desarrollo de tecnologías que pueden considerarse nichos de oportunidad para el desarrollo de productos innovadores en la región. La identificación de estos nichos de oportunidad permite elaborar estrategias en las que se estimulen la participación de diferentes actores coadyuvando a estructuras territoriales mejor integradas.

Reconocer al Distrito Federal como una fuente de innovación y de conocimiento debe entenderse, desde una perspectiva, como resultado de la concentración de diferentes industrias en un territorio específico. Así las condiciones de contexto como la inversión, los recursos humanos especializados, las fuentes de materias primas, entre otras; son elementos que estimulan las actividades inventivas.

\section{Agradecimientos}

Se agradece el apoyo de Conacyt al igual que de Francisco Javier Lozano Espinosa y a la Dirección General de Evaluación Institucional de la Universidad Nacional Autónoma de México.

\section{Referencias}

Atlas de la Ciencia Mexicana, 2012. Consultado en marzo de 2014. http://www.atlasdelacienciamexicana.org.mx.

Barroso, W., L. A. Quoniam y E. Pacheco. "Patents as Technological Information in Latin America". World Patent Information 31, no. 3 (2009): 207-215.

Batagelj, V. y A. Mrvar. Networks/Pajek64. Program for Large Network Analysis, 2 mayo de 1996. Consultado en enero de 2013. http://vlado.fmf.uni-lj.si/pub/networks/pajek/.

Cámara de Diputados. "Artículo 15", Ley de la Propiedad Industrial. México, 2006.

Conacyt. Informe general del estado de la ciencia, la tecnología y la innovación. México: Consejo Nacional de Ciencia y Tecnología, 2012.

- Sistema Integrado de Información sobre investigación Científica, Desarrollo Tecnológico e Innovación. México: Consejo Nacional de Ciencia y Tecnología. Consultado en marzo de 2014. http:// www.siicyt.gob.mx/siicyt/ 
Etzkowitz, H., A. Webster, C. Gebhardt y B. Cantisano. “The Future of the University and the University of the Future: Evolution of Ivory Tower to Entrepreneurial Paradigm". Research Policy 29 (2000): 313-330.

Guzman Chavez, A. G., F. López-Herrera y F. Venegas Martínez. "Un análisis de co-integración entre patentes y crecimiento económico en México 1980-2008”. Investigación económica 71 (2012): 83-115.

Hernández, S. y E. Díaz. "La producción y el uso del conocimiento en México y su impacto en la innovación: análisis regional de las patentes solicitadas". Análisis económico 50, no. 22 (2007): 185-217.

IMPI. IMPI en Cifras. México: Instituto Mexicano de la propiedad Industrial. 2013.

- Sistema de información de la gaceta de la propiedad industrial. México: Instituto Mexicano de la Propiedad Industrial, 2014. http:// siga.impi.gob.mx.

INEGI. Sitio web. México, 2014. Consultado en marzo de 2014. http:// www.inegi.org.mx/default.aspx.

McMillan, G. S. "Gender Differences in Patenting Activity: An Examination of US Biotechnology Industry". Scientometrics 10 (2009): 683-691.

Narin, F. “Patent Bibliometrics”. Scientometrics 30, no. 1 (1994): 147-155.

OCDE. Regional Concentration of Patents in OCDE Regions at a Glance 2009. París: OCDE, 2009. Consultado en agosto de 2013. http://dx.doi.org/10.1787/reg_glance-2009-7-en.

OCDE y Eurostat. Manual de Oslo: Guí para la recogida e interpretación de datos sobre innovación. Madrid: Trasga, 2005.

OCDE y OEPM. Manual de Estadísticas de Patentes de la OCDE. París: OCDE, 2009, 15-29.

OMPI. Concept of a Technology Classification for Country Comparisons. World Intellectual Property Organization, 2008.

—. 28 de febrero de 2014. Clasificación Internacional de Patentes. Consultado en marzo de 2014. http://cip.oepm.es/ipcpub/\#lang=es\&menulang=ES\&refresh=page

RICyT, OEA y CyTED (2001). Manual de Bogotá. Colombia, 2001.

RICyT (2009). Manual de Santiago. Buenos Aires, Argentina: RICyT, 2009.

Rodríguez-Pose, A y E. Villarreal. "Innovation and Regional Growth in Mexico 2000-2010”. Growth and Change 46, no. 2 (2015): 172195.

Sábato, J y B. Natalio. "La ciencia y la tecnología en el desarrollo futuro de América Latina”. Revista de Integración (1968).

Sánchez, Y, F. García y E. Mendoza. "Determinantes de la capacidad de innovación regional en México: Una tipología de las regiones”. Región y Sociedad 26, no. 61 (2014).

SCT. Correos de México, Consulta Códigos Postales, marzo 2014. http://www.correosdemexico.gob.mx/ServiciosLinea/Paginas/ ccpostales.aspx. 
Sugimoto, C. y R., C. Ni, J. D. West y V. Lariviére. "The Academic Advantage: Gender Disparities in Patenting”. Plos One 10, no. 5 (2015).

Toivanen, H y A. Suominen. "The Global Inventor Gap: Distribution and Equality of World-Wide Inventive Efforts, 1990-2010”. Plos One 10, no. 4 (2005).

UNAM. "Estudio comparativo de las universidades mexicanas". EXECUM, 2013. Consultado en marzo de 2014. http://www.execum. unam.mx/index2.php?_dc=0.

Verbeek, A., K. Debackere, M. Luwel y E. Zimmermann. "Measuring Progress and Evolution in Science and Technology: The Multiple Uses of Bibliometric Indicators". International Journal of Management Reviews 4, no. 2 (2002): 179-211.

Vilalta, C y H. Banda-Ortiz. "Two Hypothesis Tests for the Geography of Patents in Mexico". Economía, Sociedad y Territorio 8, no. 28 (2008): 1007-1032.

Wallin, J. "Bibliometric Methods: Pitfalls and Possibilities". Basic Clinical Pharmacology and Toxicology 97, no. 5 (2005): 261-275

Whittington, K y L. Smith. "Gender and Comercial Science: Women's Patenting in the Life Sciences". Journal of Technology Transfer 30 (2005): 355-370.

\section{Para citar este artículo:}

Meza Rodríguez, Nevid Israel, Gabriela Millán Quintero y Miguel Ángel Pérez Angón. "Patentes mexicanas del Distrito Federal: caracterización por delegación y área tecnológica (2009-2012)”. Investigación Bibliotecológica 31, no. 71 (enero-abril 2017): 181-200. DOI:http://dx.doi.org/10.22201/iibi.0187358xp.2017.71.57815 